\title{
Study on the Technology Conditions of Flue Gas Desulfurization with
}

\section{Organic Solvent}

\section{Wanren Chen ${ }^{1}$, Hua Li ${ }^{1 *}$, Xiaoshuang Chen, ${ }^{2}$}

1. School of Chemical and Energy Engineering, Zhengzhou University, Zhengzhou, Henan, China, 450001,

Email:wrchen@zzu.edu.cn

1. School of Chemical and Energy Engineering, Zhengzhou University, Zhengzhou, Henan, China, 450001,

Email: lihua@zzu.edu.cn

2. School of Energy and Power Engineering, Xi'an Jiaotong University, Xi'an, Shanxi, China, 710049,

Email: chxshuang@sina.com

\section{Abstract:}

The technology experiment on Flue Gas Desulfurization (FDG) by DMSO method is studied in this paper, and the desulfurization efficiency is set out in various conditions. The results show that the desulfurization efficiency can be over $94 \%$ under the condition as follows: the rate of flow of $\mathrm{DMSO}=100 \mathrm{~L} / \mathrm{h}, \mathrm{L} / \mathrm{G}=40 \sim 50$, absorbent concentration $=$ $100 \%$ DMSO, $T=$ room temperature, the gas flow $=60 \mathrm{ml} \cdot \mathrm{min}^{-1}$, inlet $\mathrm{SO}_{2}$ concentration $=0.1-0.5 \%$, the time of operation must be controlled in 30 minutes.

Key words: FGD, Organic solvent, SO2, desulfurization efficiency

\section{Council for Innovative Research}

Peer Review Research Publishing System

Journal: Journal of Advances in Chemistry

Vol 1, No. 1

editor@cirworld.com

www.cirworld.com, member.cirworld.com 


\section{Introduction}

Removal of SO2 from flue gas is one of very important research projects in the world. In order to find effective method, various desulfurization techniques, 1-8 such as activated carbon, molecular sieve, the electron-beam radiation technique, chemical method, physical solvents etc. are all attempted. Compared with these methods, organic solvent absorption has some advantages as low investment, high SO2 absorption efficiency and desorption efficiency and worth to further research. In this paper, based on the selected liquid organic absorbent with a high absorption and desorption efficiency with low toxicity and low price, 6 the technology experiment in various conditions on FDG by DMSO method is studied.

\section{Experimental}

\subsection{Experimental apparatus}

The experimental apparatus for removal SO2 is shown in Figure 1. The absorption apparatus of technological condition is an absorption tube with sandglass. The sulfur dioxide with a mole fraction purity $\times(\mathrm{SO} 2) \geq 0.997$, pure nitrogen with a purity $x$ (N2) $\geq 0.99999$; are both from cylinders. The sulfur dioxide and nitrogen are mixed by a gas mixer with static agitation unit to simulate the flue gas. A flowrator is used to control the flow. The SO2 analyzer with SO2 sensor (type NTS 100, Nanjing, China) was used for the $\mathrm{SO} 2$ analysis of the gas phase from the feed gas and the tail gas. The $\mathrm{SO} 2$ concentration in the gas phase before and after absorption is analyzed by $\mathrm{SO} 2$ analyzer, then the purification efficiency is calculated.

$$
\begin{aligned}
& \text { The desulfurization efficiency }\left({ }^{\eta}\right) \text { is: } \quad \eta_{=} \frac{\frac{C_{0}-C}{C_{0}} \times 100 \%}{\text { In which } \quad \mathrm{C} 0 \text { - The SO2 concentration before absorption }} \\
& \text { C- The SO2 concentration after absorption }
\end{aligned}
$$

\subsection{Material}

All the chemical reagents used are AR grade. The water is purified and deionized with a conductivity $<0.06{ }^{\mu} \mathrm{S} . \mathrm{cm}-1$.

\section{Result and discussion}

\subsection{The effect of absorbent concentration on the desulfurization efficiency}

Experiment condition: temperature: $24^{\circ} \mathrm{C}$, gas flow: $60 \mathrm{ml} \cdot \mathrm{min}-1$, inlet SO2 concentration: $0.1792 \%$. The results are shown in Table 1 and Figure 2.

Figure 2 shows that DMSO has a good desulfurization efficiency for low SO2, and desulfurization efficiency increases with DMSO concentration increase.

\subsection{The effect of absorbent temperature on desulfurization efficiency}

Experiment conditions : gas flow: $80 \mathrm{ml} \cdot \mathrm{min}-1$, inlet SO2 concentration: $0.1783 \%$, the results are shown in Table 2 and Figure 3.

Figure 3 show that the temperature influences desulfurization efficiencies. And the sulfur dioxide desulfurization efficiency decreases when the temperature increases.

\subsection{The effect of flow rate of flue gas on desulfurization efficiency}

Experiment conditions : temperature: $24^{\circ} \mathrm{C}$, inlet SO2 concentration: $0.1783 \%$. The effect results of flow rate of flue gas on 
desulfurization efficiency are shown in Table 3 and Figure 4. From Figure 4, it can be found that flow rate of flue gas influences desulfurization efficiencies, the smaller the gas flow, the higher the desulfurization efficiency. When flow rate of flue gas is $60 \mathrm{ml} \cdot \mathrm{min}-1$, the desulfurization efficiencies are over $95 \%$ within the experimented time, when flow rate of flue gas is higher $60 \mathrm{ml} \cdot \mathrm{min}-1$,the desulfurization efficiency dropped quickly.

If the gas flow is too large, SO2 was discharged with flue gas without much the mass transfer and absorption with absorption liquid, gas-liquid entrainment phenomenon will also be occurred, and lead to the decrease of the purification efficiencies. Consideration of high efficiency and economy, therefore the suitable flow rate of flue gas is selected at 60 $\mathrm{ml} \cdot \min -1$.

\subsection{The effect of liquid/gas ratio (L/G) on desulfurization efficiency}

The liquid/gas ratio directly affect rationality of investment (such as tower, pump, pipe etc.) and the running cost (such as electric power consumption etc.), is an important parameters for influencing desulfurization efficiency. The effect of L/G on desulfurization efficiency is shown in Table 4 and Figure 5. Figure 5 shows that the desulfurization efficiency increases with the increase of liquid/gas ratio, when liquid/gas ratio is higher, the absorption liquid is adequate enough, it is larger interface with SO2 from flue gas, mass transfer coefficient is larger, the absorption time is longer, therefore the desulfurization efficiency is higher. When $L / G$ is $45 \mathrm{~L} / \mathrm{m3}$, desulfuration efficiency is up to $96 \%$, Continue to increase the

$\mathrm{L} / \mathrm{G}$, the desulfuration efficiency only increase slightly, so the suitable liquid/gas ratio is selected in $45-50 \mathrm{~L} / \mathrm{m} 3$.

\subsection{The effect of inlet $\mathrm{SO}_{2}$ concentration on desulfurization efficiency}

The effect of inlet SO2 concentration on desulfurization efficiency is studied, as shown in Table 5 and Figure 6 , Figure 6 shows that the desulfurization efficiency increases with the inlet SO2 concentration increases. When the other condition was constant, the increase of the inlet SO2 concentration is equivalent to increase gaseous phase $\mathrm{SO} 2$ partial pressures, consequently, the propulsion of mass transfer between the gas and liquid phase is increased, the effect of mass transfer is heightened, the desulfurization efficiency is increased. But the extent to which desulfurization efficiency is raised decrease gradually with the inlet $\mathrm{SO} 2$ concentration increases.

\section{Conclusions}

Main parameters (absorption liquid concentration, the temperature, the gas flow, liquid/gas ratio, inlet SO2 concentration, etc.) affecting SO2 removal efficiency were studied. The results show that SO2 purification efficiency increases with DMSO concentration increase, the sulfur dioxide purification efficiency decreases when the temperature increases, the smaller the gas flow, the higher the SO2 removal efficiency, the desulfurization efficiency increases with the increase of liquid/gas ratio, the sulfur dioxide purification efficiency decreases with the inlet SO2 concentration increases.

Basing on the consideration of the experimental results and economic cost, the suitable technological conditions is: $L / G=$

$40 \sim 50$, absorbent concentration $=100 \% \mathrm{DMSO}, \mathrm{T}=$ room temperature, the gas flow=60 $\mathrm{ml} \cdot \mathrm{min}-1$, inlet SO2 concentration=0.1-0.5\%; on the above process parameters, SO2 purification efficiency can up to more than $94 \%$.

The result provided a reference for the industrial application of removal of SO2 from flue gas. 


\section{REFERENCES}

[1] Li, H., Chen, W. R.,Liu, D.Z. 2002. Study on Removing Sulfur Dioxide from Flue Gas with Liquid Absorbents. Environ. Protect. of Chem. Ind. 22(4), 193-196.

[2] Li, H., Chen, W.R. 2006. Solubility of Dilute SO2 in DMSO+Mn2+ Mixture Solvents and EOS Model-( II).Phy. and Chem. of L. 44(1), 83-93.

[3] Li, H., Chen, W.R. 2006. Solubility of Dilute SO2 in DMSO+Mn2+ Mixture Solvents and EOS Model- I. Phy. and Chem. of L. 44(2),183-191.

[4] Li, H.,Chen, W.R. 2007. Solubility of SO2, CO2 in desulfuration solution from 293.15K to $313.15 K$. Phy. and Chem. of L. 45(1), 57-65.

[5] Li, H. 2009. Thermodynamic Model of Solubility for CO2 in Dimethyl Sulfoxide. Phy. and Chem. of L. 47(3), 296-301.

[6] Li, H., Chen, W.R., Liu, D. Z. 2003. Desulfurizing Absorbent for Flue Gas and its Absorption Mechanism. J. of Environ. Sci.. 15(1), 92-96.

[7] Li, H., Chen, W.R. 2007. Solubility of SO2,CO2 in DMSO+Mn2+ Mixture Solvents and EOS Model. Phy. and Chem. of L. 45(2), 207-213.

[8]. Li, H.,Hu, G. Q.,Chen, X.S. 2010. Research on mechanism of gas liquid separation in SO2 removal from flue gas by liquid absorption with catalyzed reaction. Phy. and Chem. of L. 48(5), 652-660.

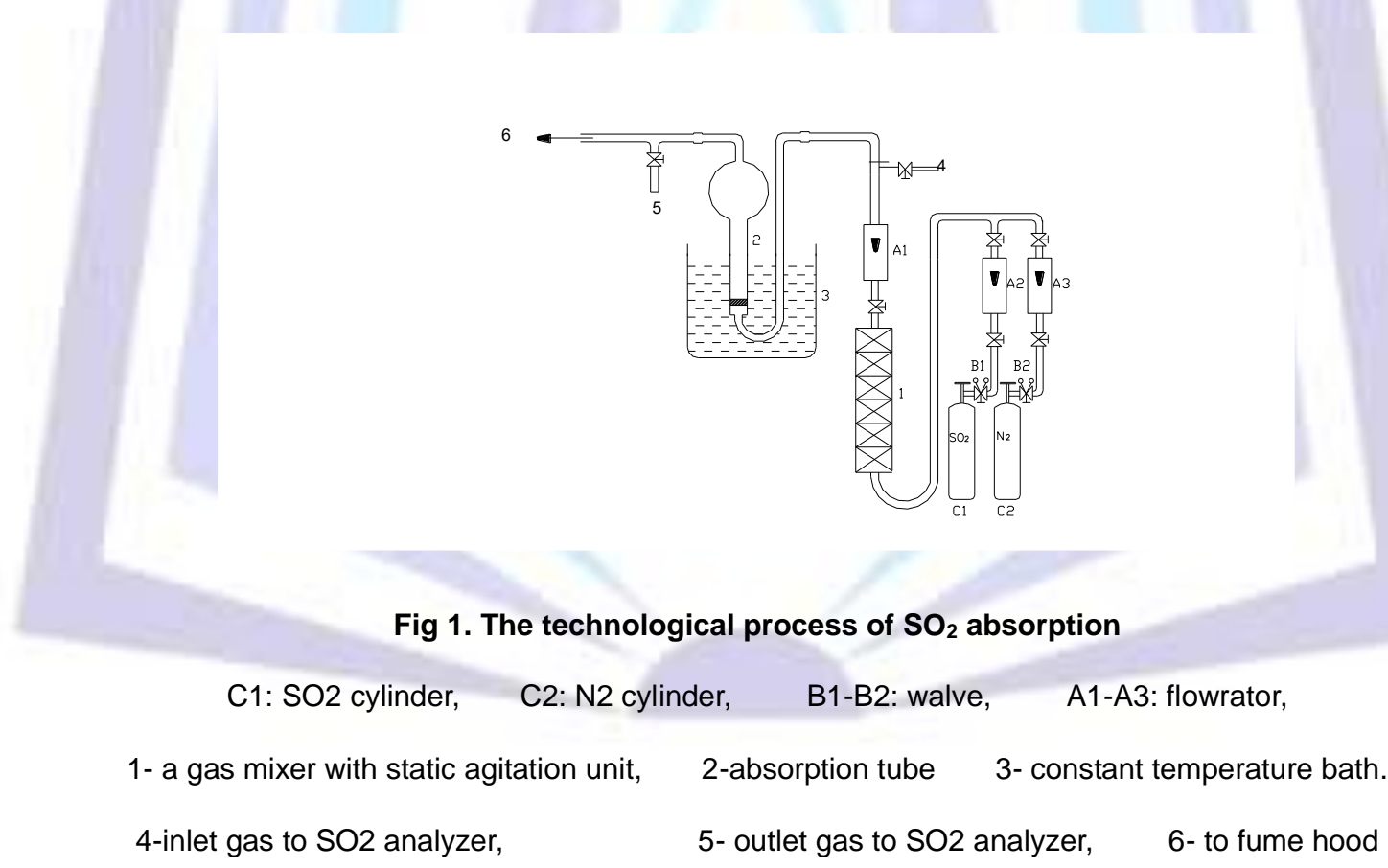




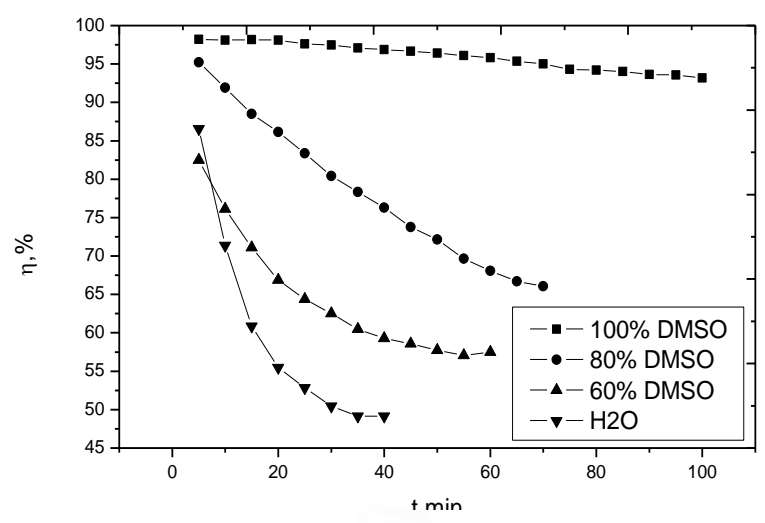

Fig. 2 The effect of absorbent concentration on desulfurization efficiency

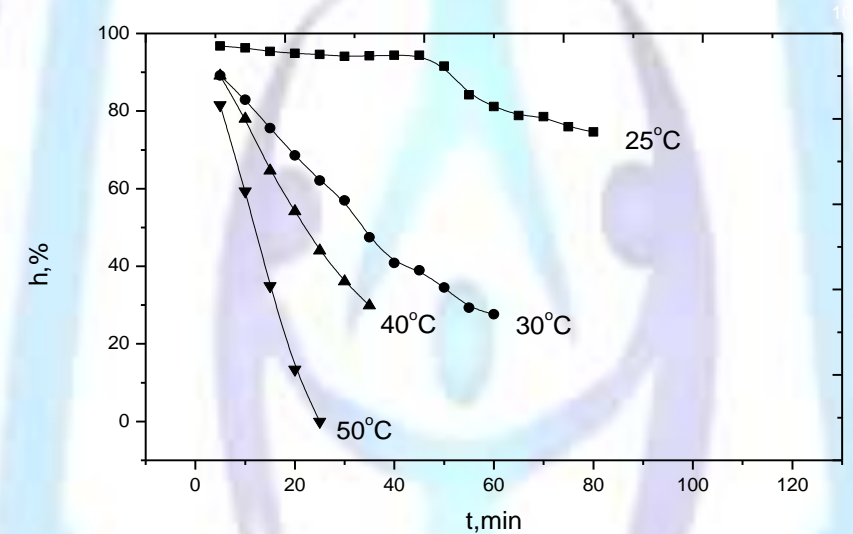

Fig. 3 The effect of absorbent temperature on desulfurization efficiency

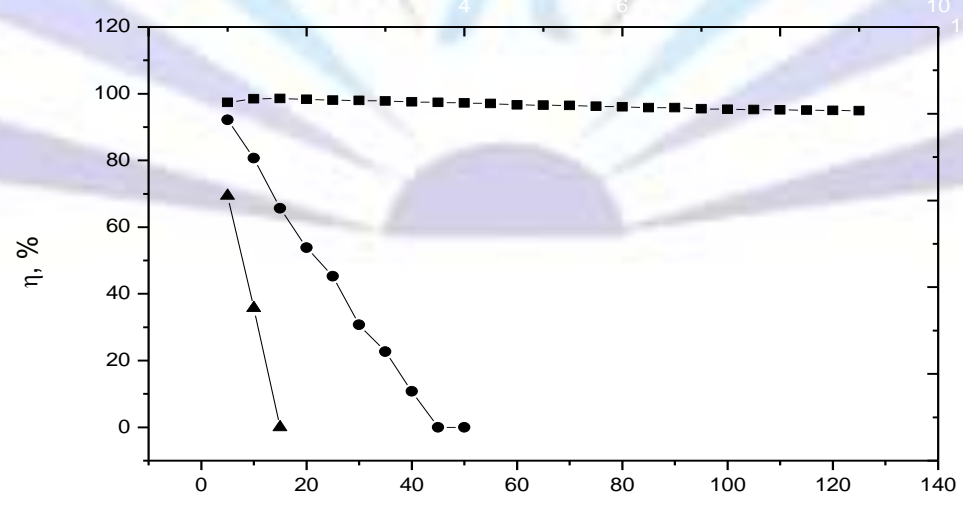

Fig. 4 The effect of flow rate of flue gas on desulfurization efficiency

घ, $60 \mathrm{ml} \cdot \mathrm{min}^{-1} ; \bullet, 100 \mathrm{ml} \cdot \mathrm{min}^{-1} ; \boldsymbol{\Delta}, 200 \mathrm{ml} \cdot \mathrm{min}^{-1}$ 


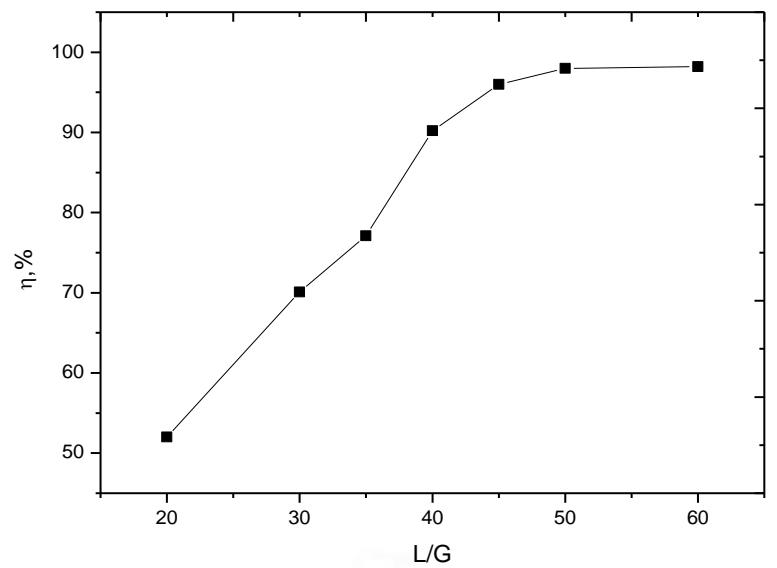

Fig. 5 Relation between $L / G$ and desulfuration efficiency

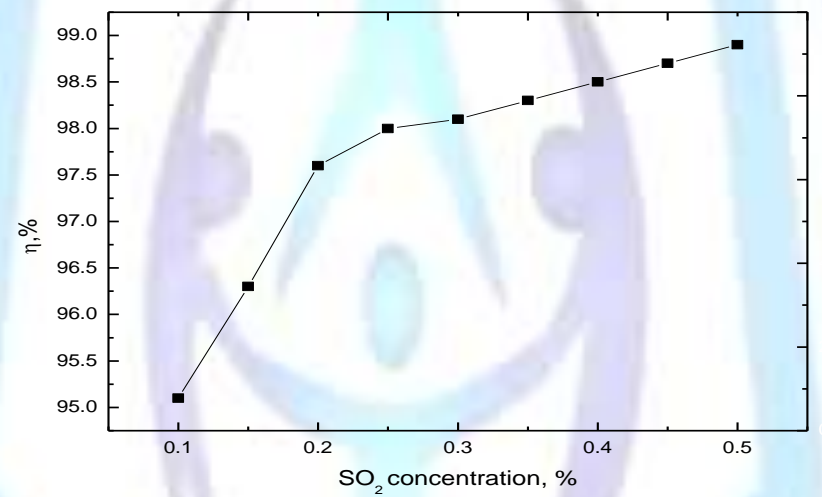

Fig. 6 Relation between inlet $\mathrm{SO}_{2}$ concentration and desulfuration efficiency

Table 1 The effect of absorbent concentration on desulfurization efficiency

\begin{tabular}{lllll}
\hline $\mathrm{t}, \mathrm{min}$ & $100 \%$ DMSO & $80 \%$ DMSO & $60 \%$ DMSO & $\mathrm{H}_{2} \mathrm{O}$ \\
\hline 5 & 98.21 & 95.20 & 82.49 & 86.57 \\
10 & 98.09 & 91.90 & 76.11 & 71.36 \\
15 & 98.15 & 88.50 & 71.08 & 60.85 \\
20 & 98.09 & 86.16 & 66.89 & 55.48 \\
25 & 97.59 & 83.37 & 64.37 & 52.85 \\
30 & 97.48 & 80.41 & 62.52 & 50.44 \\
35 & 97.09 & 78.34 & 60.45 & 49.16 \\
40 & 96.86 & 76.28 & 59.28 & 49.16 \\
45 & 96.64 & 73.77 & 58.55 & -- \\
50 & 96.42 & 72.15 & 57.71 & -- \\
\hline
\end{tabular}




\begin{tabular}{lllll}
\hline 55 & 96.08 & 69.64 & 57.04 & -- \\
60 & 95.80 & 68.08 & 57.49 & -- \\
65 & 95.35 & 66.68 & -- & -- \\
70 & 95.02 & 66.07 & -- & -- \\
75 & 94.29 & -- & -- & -- \\
80 & 94.18 & -- & -- & -- \\
85 & 94.01 & -- & -- & -- \\
90 & 93.62 & -- & -- & -- \\
95 & 93.56 & -- & -- & -- \\
100 & 93.17 & -- & -- & -- \\
\hline
\end{tabular}

Table 2 The effect of absorbent temperature on desulfurization efficiency

\begin{tabular}{lllll}
\hline $\mathrm{t}, \min$ & $25^{\circ} \mathrm{C}$ & $30^{\circ} \mathrm{C}$ & $40^{\circ} \mathrm{C}$ & $50{ }^{\circ} \mathrm{C}$ \\
\hline 5 & 96.75 & 89.18 & 89.12 & 81.55 \\
10 & 96.24 & 82.89 & 77.96 & 59.34 \\
15 & 95.34 & 75.60 & 64.61 & 34.94 \\
20 & 94.9 & 68.59 & 54.12 & 13.40 \\
25 & 94.56 & 62.09 & 44.03 & 0.00 \\
30 & 94.11 & 56.93 & 36.06 & -- \\
35 & 94.22 & 47.45 & 29.89 & -- \\
40 & 94.39 & 40.83 & -- & -- \\
45 & 94.39 & 38.98 & -- & -- \\
50 & 91.59 & 34.55 & -- & -- \\
55 & 84.18 & 29.33 & -- & -- \\
60 & 81.16 & 27.65 & -- & -- \\
65 & 78.80 & -- & -- & - \\
70 & 78.58 & -- & -- & - \\
75 & 75.94 & -- & - & - \\
80 & 74.59 & & & - \\
\hline
\end{tabular}


Table 3 The effect of flow rate of flue gas on desulfurization efficiency

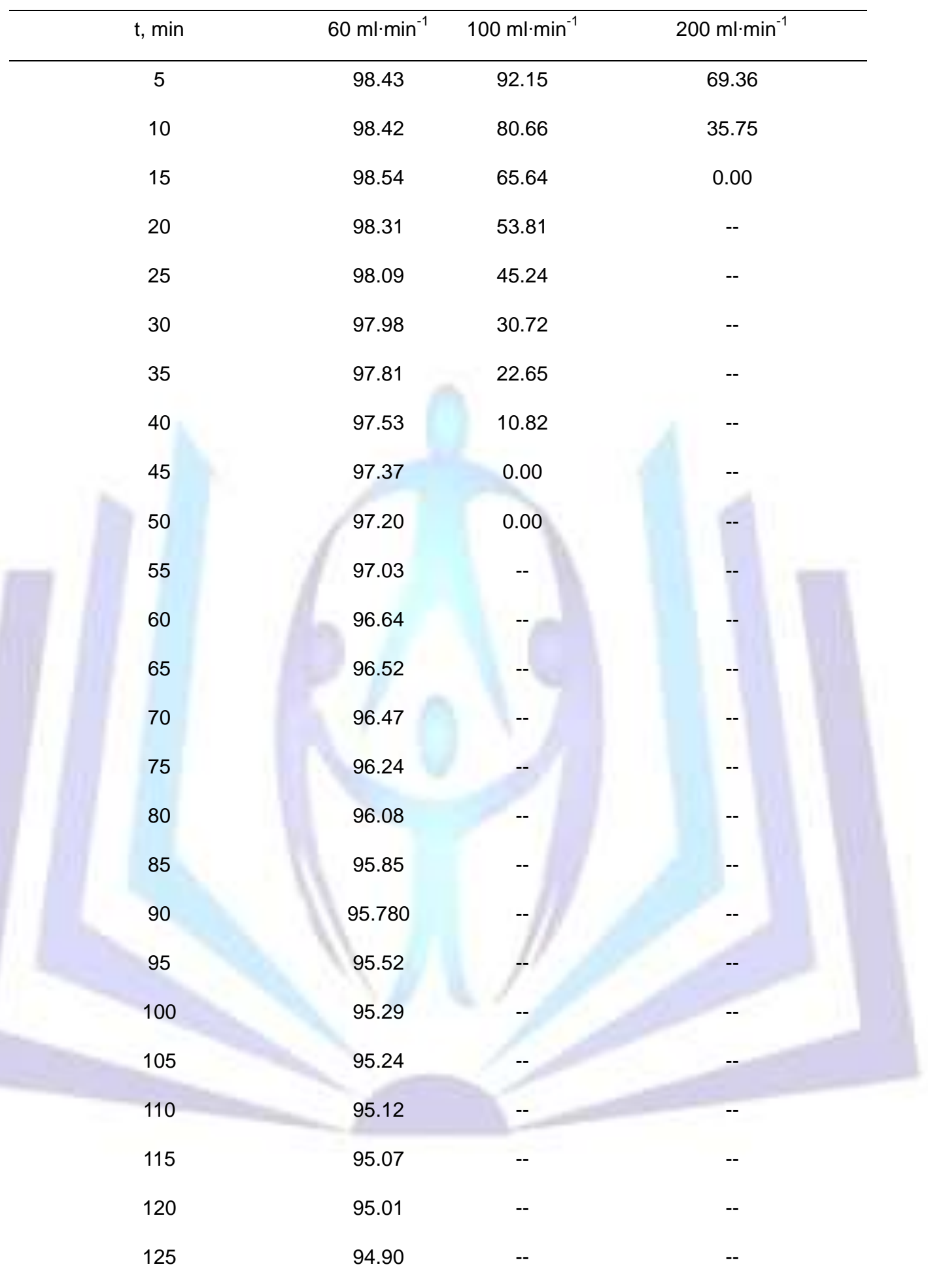


Table 4 The effect of liquid/gas ratio (L/G) on desulfurization efficiency

\begin{tabular}{cc}
\hline liquid/gas ratio, $\mathrm{L} / \mathrm{m}^{3}$ & desulfurization efficiency, \% \\
\hline 20 & 52.00 \\
30 & 70.10 \\
40 & 77.10 \\
45 & 90.20 \\
50 & 96.00 \\
60 & 98.00 \\
\hline
\end{tabular}

Table 5 The effect of inlet $\mathrm{SO}_{2}$ concentration on desulfurization efficiency

\begin{tabular}{cc}
\hline inlet $\mathrm{SO}_{2}$ concentration, \% & desulfuration efficiency, \% \\
\hline 0.10 & 95.10 \\
0.20 & 97.60 \\
0.25 & 98.00 \\
0.30 & 98.10 \\
0.35 & 98.30 \\
0.40 & 98.50 \\
0.45 & 98.70 \\
0.50 & 98.90 \\
\hline
\end{tabular}

